\title{
BioDMET: a physiologically based pharmacokinetic simulation tool for assessing proposed solutions to complex biological problems
}

\author{
John F. Graf • Bernhard J. Scholz • \\ Maria I. Zavodszky
}

Received: 10 August 2011/Accepted: 13 November 2011/Published online: 10 December 2011

(C) The Author(s) 2011. This article is published with open access at Springerlink.com

\begin{abstract}
We developed a detailed, whole-body physiologically based pharmacokinetic (PBPK) modeling tool for calculating the distribution of pharmaceutical agents in the various tissues and organs of a human or animal as a function of time. Ordinary differential equations (ODEs) represent the circulation of body fluids through organs and tissues at the macroscopic level, and the biological transport mechanisms and biotransformations within cells and their organelles at the molecular scale. Each major organ in the body is modeled as composed of one or more tissues. Tissues are made up of cells and fluid spaces. The model accounts for the circulation of arterial and venous blood as well as lymph. Since its development was fueled by the need to accurately predict the pharmacokinetic properties of imaging agents, BioDMET is more complex than most PBPK models. The anatomical details of the model are important for the imaging simulation endpoints. Model complexity has also been crucial for quickly adapting the tool to different problems without the need to generate a new model for every problem. When simpler models are preferred, the non-critical compartments can be dynamically collapsed to reduce unnecessary complexity. BioDMET has been used for imaging feasibility calculations
\end{abstract}

Electronic supplementary material The online version of this article (doi:10.1007/s10928-011-9229-x) contains supplementary material, which is available to authorized users.

J. F. Graf · M. I. Zavodszky ( $\square)$

Computational Biology and Biostatistics Laboratory, General

Electric Global Research Center, One Research Circle,

Niskayuna, NY 12309, USA

e-mail: Maria.Zavodszky@ge.com

B. J. Scholz

Pervasive Decisioning Systems Laboratory, General Electric

Global Research Center, Niskayuna, NY, USA in oncology, neurology, cardiology, and diabetes. For this purpose, the time concentration data generated by the model is inputted into a physics-based image simulator to establish imageability criteria. These are then used to define agent and physiology property ranges required for successful imaging. BioDMET has lately been adapted to aid the development of antimicrobial therapeutics. Given a range of built-in features and its inherent flexibility to customization, the model can be used to study a variety of pharmacokinetic and pharmacodynamic problems such as the effects of inter-individual differences and disease-states on drug pharmacokinetics and pharmacodynamics, dosing optimization, and inter-species scaling. While developing a tool to aid imaging agent and drug development, we aimed at accelerating the acceptance and broad use of PBPK modeling by providing a free mechanistic PBPK software that is user friendly, easy to adapt to a wide range of problems even by non-programmers, provided with readyto-use parameterized models and benchmarking data collected from the peer-reviewed literature.

Keywords PBPK modeling - Whole body model · Biodistribution - Pharmacokinetics - Mechanistic model . Imaging

\section{Introduction}

As David Leahy passionately argues in his 2004 review, complex engineering tasks these days are unthinkable without the use of computer-based simulation methods to design and test every aspect of a complex system before it is built. Similarly, the use of mathematical models of a human should be just as a standard and integral part of a pharmaceutical development process. Trial and error 
approaches are simply not viable because they are inefficient. Replacing them with rational, streamlined, and therefore more efficient design processes requires realistic and validated models [1]. Mechanistic, whole-body physiologically based pharmacokinetic (PBPK) models are the closest to a virtual human with compartments representing the organs, tissues, cells and sub-cellular compartments and with flows between them corresponding to the circulating body fluids.

The pharmaceutical industry has long recognized that physico-chemical properties determine the pharmacokinetics and pharmacodynamics of drugs [2]. Significant effort has gone into determining the ADME properties of compounds experimentally, then into developing computational tools to predict them from chemical structure alone [3]. This enabled the elimination of compounds with unfavorable properties, thus decreasing the number of failures later in the drug development process. The in vitro generated ADMET properties can be made more predictive of therapeutic outcome by incorporating them into a system model to reveal their quantitative contribution and relative importance in vivo. PBPK models are designed to integrate information about the pharmaceutical agent with the physiology properties of the host and predict the distribution of the compound in organs and tissues over time. At later stages, they can be used to optimize dosing and evaluate the performance of the agent in a diverse population. The greatest advantage of PBPK models over simpler compartmental PK models is the fact that the parameters of the former have meaning, they represent well-defined properties of the system. Because of this, it becomes possible to identify factors responsible for the undesired behavior of a compound and change the behavior by altering specific properties [4].

The environmental health field was an early adopter of PBPK modeling to assess the risk of exposure to industrial pollutants and toxins $[5,6]$. The pharmaceutical community has been slower to embrace the routine use of PBPK models. This might have been due in part to the complex nature of these models, which can require significant time and effort to implement and can be difficult to validate. The initial lack of software that could easily build and solve PBPK models likely contributed to the slow adoption of these methods within the pharmaceutical community, which instead predominantly turned to simpler modeling methods and allometric scaling rules to address their questions [7, 8]. However, several factors are now creating an environment in which PBPK models can become powerful, robust tools for the development of pharmaceuticals. First, the pharmaceutical industry is forced to look for ways to cut costs in the drug discovery and development process. The acceptance and broad application of PBPK models in early drug discovery and other phases of pharmaceutical development is one way to achieve improved productivity $[1,4,9,10]$. Second, the increasing use of imaging studies and radio-labeled drug analogues during pharmaceutical development provides an opportunity to sample multiple organ tissues rather than just blood and urine in a nondestructive manner $[11,12]$. This data can be used to test and validate PBK models. Thirdly, biological measurements are becoming more quantitative and there is an ever-increasing depth of knowledge about pathophysiology, both of which will improve the physiology parameters needed to populate the models. Lastly, the computer technology and infrastructure exists to create a flexible environment for easy implementation of PBPK models along with mechanisms for the sharing and continuous improvement of the tool by a user community.

The increasing interest in PBPK modeling is well reflected by the growing number of publications with this topic both for pharmaceutical and environmental toxicology applications [9]. A number of informative reviews have been published on recent developments and application of PBPK modeling in the preclinical and clinical phases of drug development, as well as environmental toxicology [10, 13-19]. In spite of the increase in the application of PBPK models, there remains a need for their critical and rigorous evaluation [20]. This includes assessing the predictive capacity of PBPK models with test data and clear model documentation as well as performing sensitivity, variability, and uncertainty analyses to improve the credibility and acceptance of PBPK models [5]. Our work is aimed at addressing these needs in part through its open framework for communication and sharing of the PBPK model, parameters, and test data.

The requirement for efficient pharmaceutical development exists not only in the drug industry but also in companies developing imaging agents, which share many of the same scientific and productivity challenges as pharmaceutical companies [21, 22]. Imaging agents themselves are like drugs in many ways and they are expected to have similar ADMET profiles for proper solubility, membrane permeability, etc. One important difference is that imaging agents, in order to produce a sharp image, have to reach and maintain high enough concentrations in the tissues of interest relative to the surrounding areas within the narrow time frame available for image acquisition. This makes pharmacokinetics a critical issue for imaging agents [23-26]. Another distinctiveness of imaging agent development is the need of detailed knowledge and representation of the anatomical structures to be imaged. These factors have motivated the development of our detailed, whole-body PBPK modeling tool.

In this article, we describe the physiology model and the computer implementation of the PBPK simulator that forms the core of BioDMET. The main features of the 
graphical user interface (GUI) are presented followed by the results of testing and validation. Finally, applications of BioDMET to two main areas are outlined. The tool is provided with detailed whole-body PBPK models of human, monkey, guinea pig, rat and mouse with the possibility of user-implemented adjustments for age, body weight, gender, and health condition. It also contains examples of drug/agent models as well as a validation dataset consisting of calculated biodistribution data of a number of agents in various tissues and organs compared to published experimental values. While developing a detailed PBPK tool to aid imaging agent and drug development, we strove to address the perceived gaps in the existing tools [27-42] and to accelerate the acceptance and broad use of mechanistic PBPK models by providing (1) a free, mechanistic PBPK software that can be quickly and easily adapted to the specifics of a wide range of problems even by non-programmers, (2) ready-to-use physiological and anatomical parameters for multiple species, strains, gender, and age, and (3) easily accessible test data to benchmark the predictive accuracy and confidence levels of PBPK models.

\section{Methods}

\section{The BioDMET model structure}

BioDMET enables the quick generation of complex multicompartment pharmacokinetic models that use ODEs to represent, at the macro scale, the circulation of fluid through organs and tissues, and, at the molecular scale, the biological transport mechanisms and biotransformations within cells and their organelles. This is accomplished by first defining the BioDMET model structure composed of: (1) a whole body model that includes all of the fluid spaces and their associated flows; (2) an element model comprised of the molecules, receptors, transporters, pathogens, and their interactions that are explicitly modeled, and finally; (3) the simulation setup parameters that specify the sampling time points, administration methods and starting concentrations for all the elements of interest. Once the complete BioDMET model structure is defined, the software tool automatically sets up the required pharmacokinetic compartments and the ODEs representing the flows between compartments. The ODEs are then solved to generate the concentration over time curves for the relevant elements in the model.

\section{The whole body model}

The whole body or animal model in BioDMET is a collection of fluid spaces and connections between them: surfaces and pipes with convective flows of blood and lymph. The model is hierarchical in nature where fluid spaces are contained within cells and tissues, which are components of the organ systems. All major organs are included, each being composed of one or more tissues (Fig. 1). Although some tissues have unique features, a generic tissue is composed of a vasculature space, an interstitial space, and cells (Fig. 2). Within each tissue, there are fixed tissue-specific and endothelial cells, as well as mobile blood cells. Multiple different cell types can be defined within a tissue. For example, the endocrine tissue of the pancreas (excluding the vasculature) is composed of $\sim 65 \%$ insulin secreting beta cells, $\sim 17 \%$ glucagon secreting alpha cells, $\sim 9 \%$ somatostatin secreting delta cells and $9 \%$ pancreatic polypeptide secreting cells [43]. Each cell is further divided into a number of spaces corresponding to the cytosol, endosomes, Golgi, and other organelles. The fluid in each space has its own unique set of properties including $\mathrm{pH}$, composition (e.g., water, protein, carbohydrate, lipid, DNA, RNA, mineral, gas), and the center-to-edge diffusion length characteristic of the space. While these details add complexity to the model, they are crucial to answering questions about mechanisms and feasibility assessment in the earliest stages of molecular imaging R\&D.

A space can be connected to one or more other spaces by pipes and surfaces (Fig. 1b). Pipes correspond to fluid flow between spaces and are characterized by a flow rate and the direction of flow. One example of a pipe connection is between the vasculature space of a tissue (e.g., capillary beds) and the vasculature space of the major artery or vein tissues of the cardiovascular system. Another example of a pipe connection is the one between the bile space of the liver and the bile space of the gallbladder that represents the bile ducts. Surface connections are the equivalents of membranes, such as the one between a cell's cytosol and the surrounding interstitial space. Some cells in the model are polarized, having two surfaces, which correspond to the basal and the apical plasma membranes. Examples include the epithelial cells lining the proximal tubules of the kidney, the hepatocytes of the liver, and the endothelial cells of the capillaries. The properties of a surface connection include the surface area, thickness, and composition. A surface can also have a distribution of pores that have a given dimension, charge, and fractional area of the surface. Examples of pores include the glomerular pores in the endothelial cells of the kidney. Another example of a surface with pores is the nuclear membrane.

The parameters for the whole body model were defined to facilitate easy scaling of the model's volumes and flows. While each tissue has an absolute volume, the cells and fluid spaces contained within the tissue are defined with a 
Fig. 1 a The BioDMET whole body model is a hierarchical structure of all major organs and organ systems (green hexagons), each composed of one or more tissues (orange quadrangles). b The tissues are made of cells (purple octagon) and spaces (blue rectangle). The spaces are connected by pipes (magenta lines) representing blood or lymph flow and by surfaces (blue lines). The arrows on the magenta lines show the direction of the pipe flow. A dotted line means that the space at one end of the connection is collapsed in the hierarchical view. When a space is selected at the GUI (Interstitial currently), its connections to the rest of the system are automatically shown
A

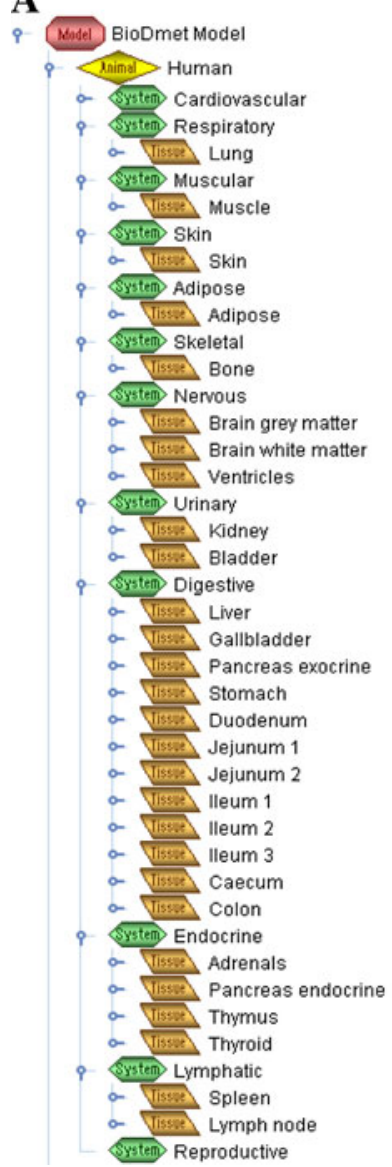

B

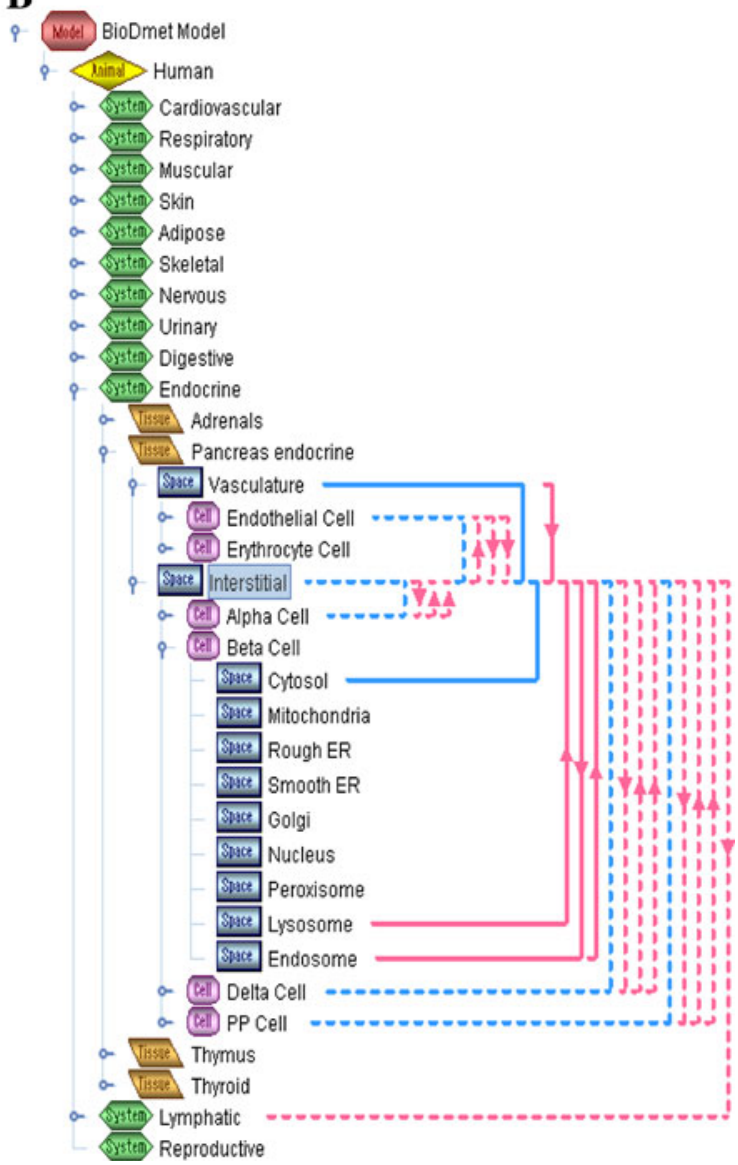

Fig. 2 Tissue and cell structure with the types of transport processes modeled in BioDMET

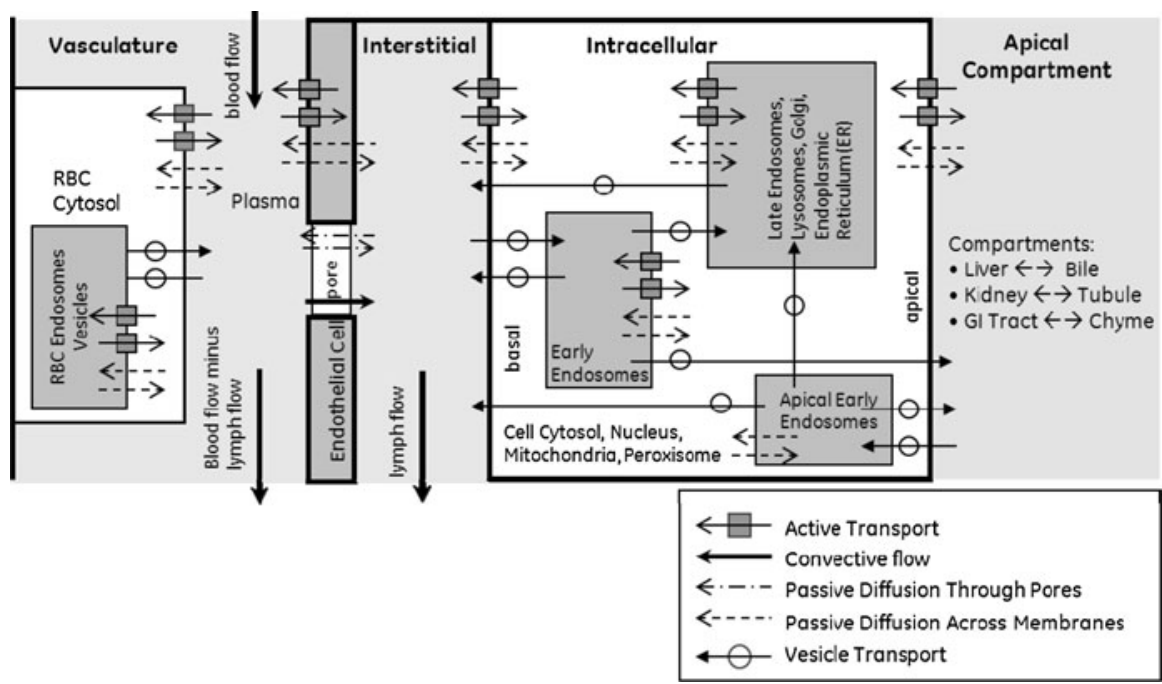

volumes and masses for the tissues, cells, and fluids of the body.

The flow rates between spaces have also been set up to allow easy scaling of a model. For example, the arterial blood flow to a tissue is defined in terms of volume of flow per time per volume of tissue, while the venous and lymph flows of the tissue are defined relative to the arterial blood 
flow. Thus changing the tissue volume triggers the automatic scaling of the tissue's blood and lymph flow in the correct proportions. The flow of the chime, bile, pancreatic juice, or CSF is defined in a similar manner. However, the software tool allows the user to override any of the default or scaled parameter values of the whole body model.

\section{The element model}

Elements within BioDMET are defined to explicitly model molecules, receptors, transporters, and even pathogens within the whole body model (Fig. 3). Each element type or class has a set of properties to define its behavior. The agent administered to the animal is perhaps the most important element of the model. The agent can be composed of one or multiple molecules (e.g., a drug and its metabolites). BioDMET has been used to study the biodistribution of molecules that range in molecular weights from small molecules $(<1 \mathrm{kDa})$ to medium peptides $(1-60 \mathrm{kDa})$ to large proteins $(>60 \mathrm{kDa})$ and particles. The size of the molecule changes its permeability and clearance characteristics as determined by the physics-based equations in the model. Other relevant physico-chemical properties include the molecule's charges and $\mathrm{pH}$ compartmental effects (captured by the $\log \mathrm{D}$ ), which influence its membrane permeability and tendency to partition into lipid or aqueous spaces. The plasma protein binding and liver microsomal clearance rate are additional properties that are required as input.

Receptors, transporters, and biotransformation reactions are also part of the element model. They can be included by specifying enzyme concentrations, sub-cellular locations, substrates, and kinetic rates. The tool makes it possible to calculate the influence of the competitive effects of receptor saturation and molecular transport on a molecule's biodistribution. This can be done for both endogenous substances and metabolites of the parent molecule. The capability to model multiple metabolites of the parent molecule has been used to understand the loss in imaging contrast in specific tissues. It can also be used to model metabolite-induced toxicity of drugs.

Elements can be set up to interact with each other such as the binding between a molecule and a receptor. Another example of an element interaction is the conversion of one molecule into two other molecules (e.g., cleavage) by a biotransformation enzyme.
Fig. 3 The components of the BioDMET Model: Animal, Elements, Administer, and Sampling. The element model defines the molecules, receptors, transporters, and their interactions that are explicitly modeled. The green lines show the interactions between elements and their locations within the animal model. Pink lines are for the injection properties. A dotted line means that the space for one end of the connection is collapsed in the hierarchical view. Only the interactions for the current selected element (GLP-1 Binder is currently selected) are shown at a given time

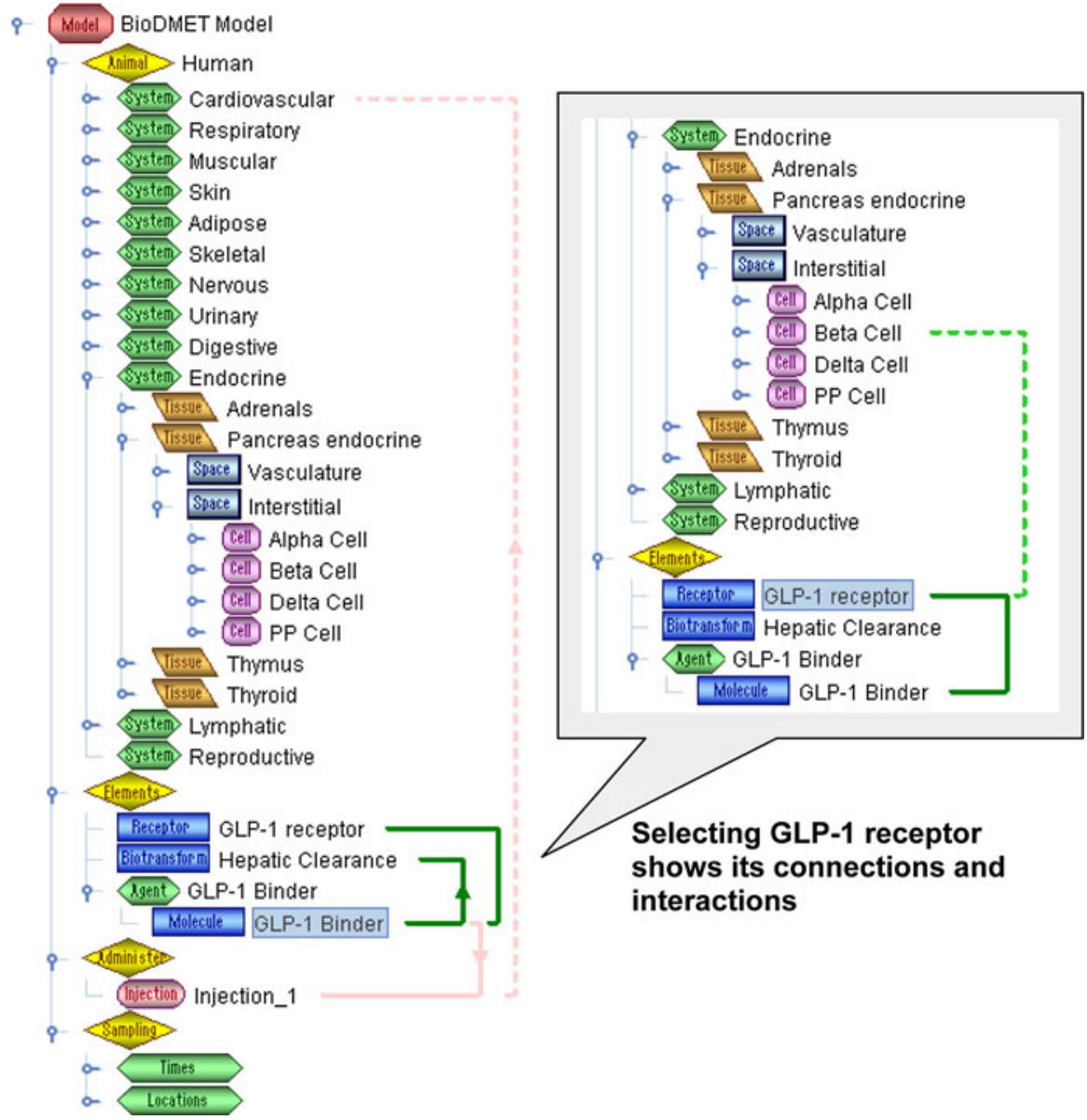




\section{Simulation run parameters}

Information about the dose, administration method (oral, intravenous, subcutaneous, or intramuscular), number of administrations, and sampling time points are captured under the Administer and Sampling components of the model (Fig. 3). In addition to the existing standard tissue spaces, a set of sampling locations are defined by merging several anatomical spaces and averaging the simulated element concentrations in them. For example, the sapling location Brain includes the white matter, the grey matter and the ventricles. The sampling location Small intestine includes the duodenum, the two segments of the jejunum and the three segments of the ileum. User-defined sampling locations are also enabled.

Initializing the PBPK compartmental model and ODEs

Besides the spaces and elements described above, BioDMET utilizes the concepts of entity and compartment in ways that might differ from the traditional PK nomenclature. An entity is a discrete unit, which, at least in theory, is distinguishable and measureable. If a molecule and its receptor are defined elements of the current model, there are a total of three entities: the unbound molecule, the free receptor, and the bound molecule-receptor complex. A compartment is defined as the unique pair of an entity and a fluid space. In theory, an entity could be present in any of the fluid spaces defined in the whole body model. Thus if the BioDMET model has 3 defined entities and 789 fluid spaces, there could be 2,367 unique compartments. However, due to various barriers to transport and interactions between entities, the entity concentration is zero in a large number of the compartments. For example, if the receptor of a molecule is defined to be present in the beta cell membranes of the endocrine pancreatic tissue, that receptor and the corresponding molecule-receptor complex will have zero concentrations in all other spaces. At the beginning of each simulation, the software generates a list of all possible entities, flow equations, and compartments. It then determines which compartments could have nonzero entity concentrations at some time point of interest. These compartments and their corresponding flow equations define the full PBPK model for that particular simulation.

In order for the tool to be non-restrictive in its scope and ready to use for a variety of projects, the BioDMET model must be detailed in the compartments that are predefined with their physiological parameters available in the database (Figs. 1,2). This richness in details comes at a cost of computational time when working with hundreds of compartments. We have devised a method and algorithm that allows some compartments to be collapsed and approximated when they do not significantly impact the results of the simulation. The collapsing of compartments is done just prior to submitting the BioDMET PBPK model to the ODE solver. At the end of the calculation, the solver results are translated (expanded) back to the rich compartment model view. This collapsing process is not visible to the user although it is possible to customize what compartments are collapsed at the GUI.

\section{Model equations}

Hundreds of differential equations have to be solved to simulate the biodistribution of a molecule in a BioDMET whole body model. All of these differential equations are associated with a limited number of event types such as the administering of a molecule to a fluid space, binding of the molecule to a receptor, biotransformation of the molecule into another form, and transport of the molecule from one fluid space to another. To provide some illustrative examples, the differential equations describing several modes of transport are presented below.

The simplest form of transport is the convective flow of an entity between two fluid spaces connected by a pipe. An example is the vasculature space of the main arteries with the vasculature space of a tissue. Assuming flow occurs from space 1 to space 2, the change in concentration of an entity $\left(C_{1}\right.$ and $\left.C_{2}\right)$ per unit time in the two fluid spaces is a function of the flow rate $\left(\mathrm{J}_{1 \rightarrow 2}\right)$ and the volumes of the spaces $\left(\mathrm{V}_{1}\right.$ and $\left.\mathrm{V}_{2}\right)$ as described by Eqs. 1a and $1 \mathrm{~b}$.

$$
\begin{aligned}
& \frac{d C_{1}}{d t}=-\frac{J_{1 \rightarrow 2} C_{1}}{V_{1}} \\
& \frac{d C_{2}}{d t}=\frac{J_{1 \rightarrow 2} C_{1}}{V_{2}}
\end{aligned}
$$

A more complex form of convective flow occurs through pores that may limit the flow of an entity based on its size. For example, the pores found in the kidney glomerular capillaries act as a filter for the fluid that flows from the vasculature space through the fenestrated endothelial cells of the glomeruli and into the lumen space of the renal tubules. In BioDMET, this type of situation is modeled by connecting the two fluid spaces, the capillary vasculature space and the renal tubule lumen space with both a pipe and a surface connection. The pipe defines the convective flow from the one space to the other and the surface connection defines the type, size, and fractional area of the pores of the fenestrated endothelial cells. Equations $2 a-2 c$ describe this filtered convective flow process. Note that the total convective flow, $\mathrm{J}_{1 \rightarrow 2}$, is multiplied by a summation term over all pores. The summation term includes the pore's fractional surface area, $\phi_{\mathrm{i}}$, and the reflection coefficient, $\sigma_{\mathrm{i}}$, of the entity trying to pass through the 
pore. The reflection coefficient is characteristic for each entity and pore combination, and is mainly dependent upon the entities' hydrodynamic radius, $\mathrm{R}_{\mathrm{h}}$, and the pore radius, $r_{\text {pore }}$ (Eq. 2c). This relationship can be used when there are no charge-charge interactions or they are negligible [44].

$$
\begin{aligned}
& \frac{d C_{1}}{d t}=-\frac{\left(J_{1 \rightarrow 2} \sum_{i=1}^{\text {pores }} \Phi_{i}\left(1-\sigma_{i}\right)\right) C_{1}}{V_{1}} \\
& \frac{d C_{2}}{d t}=\frac{\left(J_{1 \rightarrow 2} \sum_{i=1}^{\text {pores }} \Phi_{i}\left(1-\sigma_{i}\right)\right) C_{1}}{V_{2}} \\
& \lambda=\frac{R_{h}}{r_{\text {pore }}}\left\{\begin{array}{l}
\lambda<1 \quad \sigma=1-\frac{(1-\lambda)^{2}\left(2-(1-\lambda)^{2}\right)\left(1-\frac{\lambda}{3}\right)}{1-\frac{\lambda}{3}+\frac{2 \lambda^{2}}{3}} \\
\lambda \geq 1 \quad \sigma=1
\end{array}\right.
\end{aligned}
$$

A third type of flow is the passive diffusion of an entity from one fluid space to another by crossing a surface. A classic example is the passive diffusion of a molecule from the interstitial space of the tissue into the cytosol of the cell across its plasma membrane (Eqs. 3a-3f). The concentration changes in the two neighboring spaces depend on the permeability rates $\left(P_{1 \rightarrow 2}, P_{2 \rightarrow 1}\right)$, the partition coefficient $\left(\mathrm{K}_{\mathrm{p}}\right)$, and the surface area separating the spaces $\left(\mathrm{S}_{12}\right)$. Note that for neutral molecules, the permeability rate is the same in both directions $\left(P_{1 \rightarrow 2}=P_{2 \rightarrow 1}\right)$. The partition coefficient $\mathrm{K}_{\mathrm{p}}$ (Eq. 3c) is a function of the volume fractions of the aqueous phase $\left(\phi_{\text {aqueous,1 }} \phi_{\text {aqueous,2 }}\right)$ and organic phase $\left(\phi_{\text {organic,1 }}\right.$ $\phi_{\text {organic,2) }}$ of both spaces. The $\operatorname{LogD}$ describes how the entity partitions between an organic and aqueous phase of equal volume (Eq. 3d). For neutral molecules the $\log D$ is the same as the $\log P$. For ionizable molecules, however, the $\log D$ depends on the $\mathrm{pH}$ of the two fluid spaces. The permeability rate of the entity from the fluid space one to two $\left(\mathrm{P}_{1 \rightarrow 2}\right)$, is a function of the average diffusion rate out of fluid space one, $\mathrm{P}_{1}$, across the surface $\mathrm{P}_{12}$, and into fluid space two, $\mathrm{P}_{2}$ (Eq. 3e). The overall permeability rate from space one to two depends on the average distance of travel, $\mathrm{d}_{1}, \mathrm{~d}_{12}, \mathrm{~d}_{2}$ (defined in the BioDMET whole body model for each space) and the diffusion coefficients $D_{1}, D_{12}, D_{2}$ of the entity in the two fluid spaces and the separating surface. Assuming the surface is a lipid membrane, the $\log D$ is used to approximate the probability for a molecule to go from a generally aqueous environment into one that is almost entirely organic (Eq. 3e). The diffusion coefficient for each environment is computed using the Stokes-Einstein equation (Eq. 3f) as a function of the entities' hydrodynamic radius $\left(R_{h}\right)$, the viscosity and the temperature of the fluid $\left(\eta_{1}, \mathrm{~T}\right)$, and Boltzmann's constant $(\varepsilon)$. $\frac{d C_{1}}{d t}=\frac{P_{2 \rightarrow 1} S_{12} C_{2}}{K_{p} V_{1}}-\frac{P_{1 \rightarrow 2} S_{12} C_{1}}{V_{1}}$

$\frac{d C_{2}}{d t}=\frac{P_{1 \rightarrow 2} S_{12} C_{1}}{V_{2}}-\frac{P_{2 \rightarrow 1} S_{12} C_{2}}{K_{p} V_{2}}$

$\frac{1}{K_{p}}=\frac{\Phi_{\text {aqueous }, 1}+10^{\log D\left(p H_{1}\right)} \Phi_{\text {organic }, 1}}{\Phi_{\text {aqueous }, 2}+10^{\log D\left(p H_{2}\right)} \Phi_{\text {organic }, 2}}$

$\log D=\log \left(\frac{C_{\text {organic }}}{C_{\text {aqueous }}}\right)$

$P_{1 \rightarrow 2}=\frac{1}{\left(\frac{1}{P_{1}}+\frac{1}{P_{12}}+\frac{1}{P_{2}}\right)}=\frac{1}{\left(\frac{d_{1}}{D_{1}}+\frac{d_{12}}{10^{\log D\left(p H_{1}\right)} D_{12}}+\frac{d_{2}}{D_{2}}\right)}$

$D_{1}=\varepsilon \frac{T}{6 \pi \eta_{1} R_{h}}$

Clearance mechanism

Agents are generally eliminated from the body through biliary and/or renal excretion either directly or after being metabolized. For renal clearance, the agent's excretion rate is dependent upon the rate at which it is passively filtered through the kidney's fenestrated glomerular capillaries and the rates of secretion and reabsorption across the kidney tubular epithelium. Within BioDMET, the passive filtration through the fenestrated glomerular capillaries is modeled as a filtered pipe (Eq. 2). The convective flow of the pipe, $J_{l \rightarrow 2}$, is equivalent to the glomerular filtration rate (GFR) that depends on the blood flow to the kidney $\left(\mathrm{Q}_{\mathrm{kidney}}\right)$ and the hematocrit (Ht) as described by Eq. 4a. The glomerular capillaries of the human model have $8.2 \mathrm{~nm}$ diameter pores [45]. Because of the negative charges lining the surfaces of the pores, agents with various charges will pass through the pores at different rates for a given hydrodynamic volume. The function used to compute the agent's reflection coefficient (Eq. 2c) for neutral agents was empirically derived based on the effects of size and electrical charge of dextran on its filterability by the glomerular capillaries [45]. The plasma protein binding of the agent is taken into consideration when modeling the filtration process. By default, active secretion in the proximal tubules and reabsorption in the distal tubules of free agents are not accounted for. However, the model can be easily customized to include these processes once the rates are known or can be estimated. Albumin-bound agents are reabsorbed together with albumin which is filtered through the pores of the glomeruli and then are partially reabsorbed through the epithelial cells lining the proximal tubules [46, 47].

For agents/drugs that are metabolized by the liver, BioDMET models hepatic clearance using in vitro measured microsomal clearance rates $\left(\mathrm{Cl}_{\text {microsomal }}\right)$, although 
alternative inputs (parent molecule half life $\mathrm{T}_{1 / 2}$, liver tissue clearance rate $\mathrm{Cl}_{\text {liver }}$, or hepatocyte clearance $\mathrm{Cl}_{\text {hepatocyte}}$ ) are also accepted. The relationships between the microsomal clearance rate and the other measures of liver clearance are defined by Eqs. $4 b-4 d$, where $V_{\text {incubate }}$ is the incubation volume, $\mathrm{m}_{\text {microsomes }}$ and $\mathrm{n}_{\text {hepatocyte }}$ refer to the amount of microsomes and the number of hepatocytes per gram of liver tissue, respectively. The in vivo rate of liver metabolism $r$ is calculated from the in vitro liver microsomal clearance rate taking into account the mass of the liver (M), the volume of the space $(\mathrm{V})$ where the metabolic reactions take place, and the conversion factor ( $\mathrm{f}=45 \mathrm{mg}$ microsomal proteins $/ \mathrm{g}$ of liver [48]) according to Eq. 4e.

$$
\begin{aligned}
& G F R=0.2 \cdot(1-H t) \cdot Q_{\text {kidney }} \\
& T_{1 / 2}=\frac{0.693 \cdot V_{\text {incubate }}}{C l_{\text {microsomal }}} \\
& C l_{\text {hepatocyte }}=C l_{\text {microsomal }} \cdot \frac{m_{\text {microsome }}}{n_{\text {hepatocyte }}} \\
& C l_{\text {liver }}=C l_{\text {microsomal }} \cdot m_{\text {microsome }} \\
& r=C l_{\text {microsomal }} \frac{f \cdot M}{V}
\end{aligned}
$$

The tool allows the user to select either a first order or a Michaelis-Menten type reaction for describing liver metabolism. Since in vitro measured liver microsomal or hepatocyte clearance rates characterize the overall disappearance of the compound in a liver preparation (including multiple possible metabolic processes as well as diffusion through membranes) and not just the rate of the individual enzymatic reactions inside the liver cells, the place of this biotransformation in BioDMET is the interstitial space of the liver.

\section{Model parameters and data sources}

To populate the physiology models, parameter values have been obtained from the published literature on mice, rats, guinea pigs, monkeys, and humans including some specifics on strains, age, gender, and body mass index (BMI). The type of data that has been collected includes: mass/volume of tissues and size of cells; cardiac output and flow rates of blood, lymph, bile, chime, urine; surface areas of cellular membranes including small intestines, proximal tubule epithelial cells of the kidney and hepatocyte canaliculi of the liver; water/organic phase content of vascular, interstitial, and sub-cellular compartments; degree of capillary fenestration for the different organ tissues; distances of travel within vascular, interstitial, and sub-cellular compartments.

Every parameter defined in a BioDMET model represents something that can be defined physically and can be measured independently. For example, each fluid phase has a defined composition of water, protein, carbohydrate, lipid, DNA, RNA, mineral, and even air (e.g., alveolus in lung), allowing the computation of the relative volumes of the organic and aqueous phases of every space. This, in turn, is used when computing how a molecule may prefer one fluid space over another based on the molecules hydrophobic/hydrophilic characteristics as described by its $\log \mathrm{P} / \operatorname{LogD}$. The alternative approach is to use tissue partitioning coefficient derived by fitting the model to experimental in vivo data for each species of interest.

The number and size of clefts and pores between the endothelial cells are parameters defined for each tissue's capillary bed [44, 49-54]. The pores and clefts of the capillaries have a significant impact on the vascular-interstitial permeability for highly charged or moderately high molecular weight molecules. The alternative approach would be to use a tissue permeability coefficients derived by fitting the model to experimental in vivo data. This empirical approach becomes more difficult since the permeability behavior is dependent upon multiple flow processes across or between the endothelial cells of the capillary wall. Because BioDMET defines the base attributes such a fluid composition, distances between fluid spaces, pore/cleft surface area and size, more mechanistic flow equations can be derived. Furthermore the base attributes can be independently measured and derived and do not rely on fitting the model to in vivo data.

In addition to the parameters described above, all relevant physico-chemical properties for the agent (molecular weight, $\log$ P, $\operatorname{LogD}$ as a function of $\mathrm{pH}$ ) are to be specified by the user when setting up a simulation. The hydrodynamic radius is calculated based solely on the molecule's physico-chemical characteristics. The diffusion coefficient is determined using the Stokes-Einstein equation in water and at body temperature. Corrections are then used to compute a diffusion coefficient for the interstitial fluid and the membrane. These corrections are estimates based on fitting to experimental values of permeability for artificial membranes and isolated perfused tissues [55, 56].

Biochemical properties have to be provided as well, including enzymes that the molecule is a substrate for and possible metabolites of the parent compound with their properties. The ability to track the metabolites besides the parent compound is especially important for imaging since the radioactive label usually remains attached to one of them and will influence the generated image. The term enzyme in the model is used to describe a protein that can bind, transport, or catalyze the transformation of the substrate molecule. The kinetic rate parameters for the enzyme-molecule complex are also expected as part of the input. 
Fig. 4 Overview of the software components and the simulation process

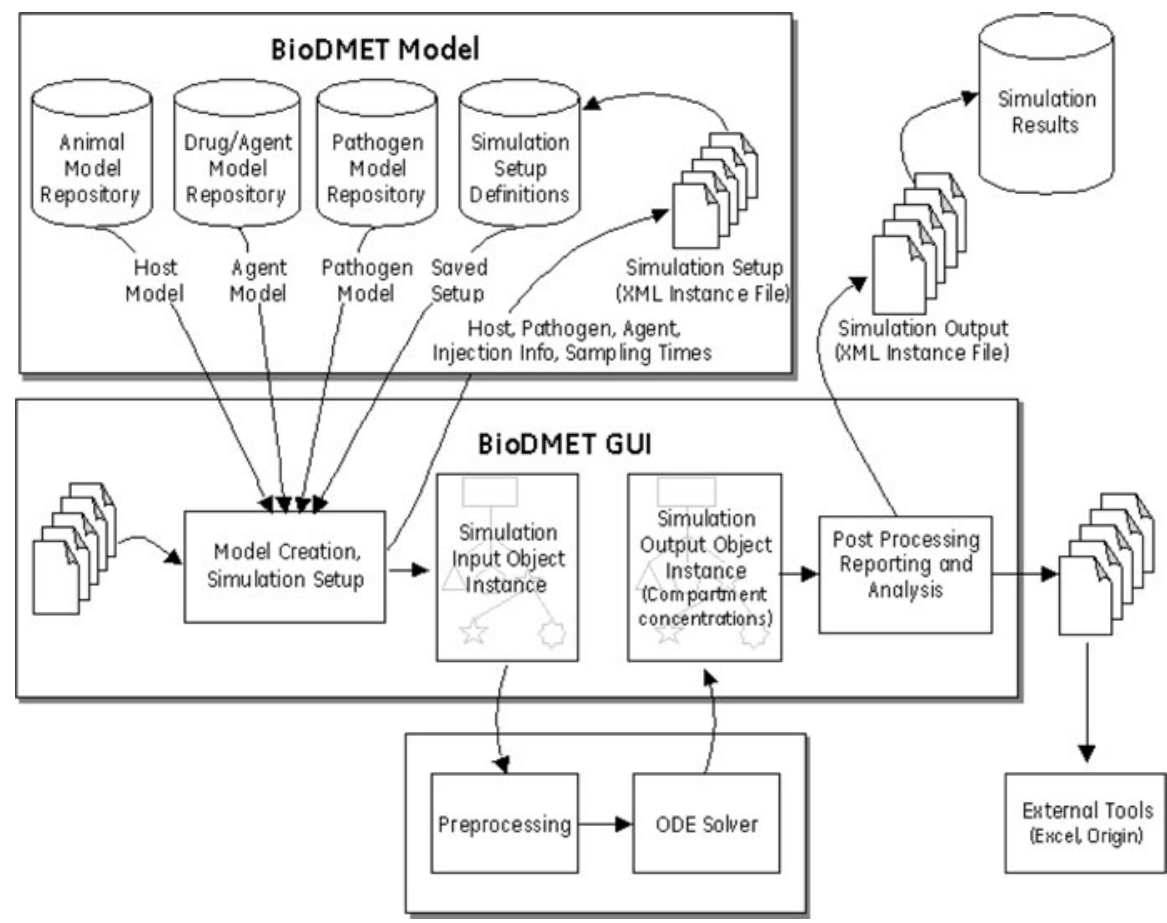

\section{Computer implementation}

The BioDMET software tool is composed of three major parts (Fig. 4):

1. A PBPK model (BioDMET Model) with a database of host, agent, and pathogen models that can be extended as necessary and incorporated by users into their simulations.

2. A model simulator built around an ODE solver.

3. A GUI used to define, run, and view results of the PBPK simulator.

\section{The BioDMET database}

The BioDMET software is bundled with a set of existing host, agent, and pathogen models. These models are prebuilt components that can be used to set up a simulation. They are stored as files in an XML-compliant markup language, which was chosen in order to facilitate interchange of the model data with other systems/applications. The data contained in the XML model files are read directly by the software. At minimum, a BioDMET run requires a host file and an agent file as the input for a biodistribution calculation. For a pathogen-host simulation, an additional pathogen model file is required. The agent file holds all relevant physico-chemical property information for the exogenous molecule(s) being studied, such as molecular weight, $\log \mathrm{P}$, and $\log \mathrm{D}$ as a function of $\mathrm{pH}$. This file also contains biochemical properties of the molecule, if available, including possible metabolites of the parent and their properties as well as known enzymes that the molecule is a substrate for. The term enzyme in the model is used to describe a protein that can bind, transport, or catalyze the transformation of the substrate molecule. The kinetic rate parameters for the enzyme-molecule complex are specified in this file. The host file holds all the anatomical, physiological, and cellular parameter data for the animal or human. All of this information is independent of the molecule whose biodistribution is studied. This host file can also hold the names, concentration, and sub-cellular locations of the enzymes for binding, transporting, or catalyzing a biotransformation. The existing host models reflect the physiological state of the healthy organism. However, a disease can significantly alter the values of these parameters, influencing the distribution of exogenous agents. Therefore, we developed a database of physiology parameters for the critically ill and implemented the capability to model several disease states such as severe burns, multiple organ failure, etc.

\section{$P B P K$ simulator and $O D E$ solver}

As described above, the tool uses ODEs to represent at the macro scale the circulation of fluid through organs and tissues, and at the molecular scale the biological transport mechanisms and biotransformations within cells and their organelles. The BioDMET GUI passes the Simulation Input Object Instance on the PBPK Model Simulator. At its heart, the simulator contains an ODE solver. 
Prior to running the ODE solver, preprocessing of the input object structure is performed, including validation of the input parameters for correctness and completeness. As the simulation runs, the output data augments the input object instance structure. This can be thought of as a new object instance structure, called the Simulation Output Object Instance (Fig. 4). The simulation itself calculates concentrations of the agent in each of the sampling locations and at each of the sampling times defined by the simulation input parameters. This information is stored along with the original input structure as the output object instance. Once the simulation completes, this structure is passed back to the BioDMET GUI tool.

\section{GUI}

One of the major components of the BioDMET application is the GUI, which can be used to create host, agent, and pathogen models as well as setup and configure PBPK simulations. The tool allows users to choose from any of the host or pathogen models stored in the Model Database, import them into the tool and modify as needed.

The GUI also provides some basic analysis with means of graphing simulation results-plots of agent concentrations over time at the various sampling locations in the host model anatomy. The user can also export the measurement data to external tools such as Microsoft Excel. Furthermore, the output of the simulation can be saved in XML form on the user's file system. This data can be used as input to other applications for post processing, analysis, and reporting. Simulation results from previous runs can be reloaded into the BioDMET GUI tool, modified as needed, and then rerun for comparative analysis.

There are three main sections to the user interface: the model tree view-displaying the simulation configuration information in a hierarchical view, the property windowwhich displays property sheets for the currently selected item(s) in the hierarchy display, and the information window-which provides access to additional information on the simulation setup and results (Fig. 5).

Setting up and running a simulation

\section{The website}

BioDMET has a web site (https://pdsl.research.ge.com/ BioDMET/) hosted by GE Global Research that allows easy access to the latest release of the tool, tutorials, documentation and nonproprietary models, test data, notes and references (Supplementary Fig. S1). Users can run the application via a standard web browser over the Internet. Access to the program is granted after a one-time registration requiring the user to provide a name, affiliation and e-mail address. Currently, all user-generated models and data are stored on the client's computer disk storage and NOT on the GE hosting server.

\section{Wizards}

Setting up a PBPK simulation with BioDMET involves multiple steps including constructing the host model hierarchy, creating the pipe and surface connections between the spaces in the hierarchy, defining the enzyme and pathogen types and their concentrations in the appropriate spaces in the host model, specifying the agents to be administered along with the method of administration and the amount being administered, the sampling time points and locations, as well as defining a pharmacodynamic function with its necessary parameters. This can be a daunting task. To make it easier, BioDMET includes a number of pre-defined host/ species, agent, and pathogen models which can be added to the simulation setup and customized as needed. In addition, several wizards have been designed to guide the user through the process of creating a new pathogen model, setting up a new biodistribution (PK) simulation or a new pathogen-host (PK-PD) simulation, performing sensitivity analysis or a critically-ill patient analysis. The wizards allow a user to define a new simulation more-or-less from scratch without having to possess a mental map of the simulation setup and without being aware of the fine details of the host's physiological model structure. During this guided process, the user can import and modify existing host/species, agent, and/or pathogen models from the BioDMET model database. Alternatively, new hosts, agents, and/or pathogens can also be built. In either case, the wizard guides the user through the process of defining the key parameters for the simulation in a step-by-step fashion (Supplementary Fig. S2). Detailed step-by-step tutorials can be found at the BioDMET web site under User Guide, which provide examples for going through the wizards for setting up and running the different simulation types implemented in BioDMET. In addition, all functionalities of the software are described in the user manual accessible on the above-mentioned web site.

Create a new pathogen model This wizard does not actually result in the creation of a new simulation. Rather, it allows a user to define a new pathogen model and save it to a file on the file system. The pathogen model file can subsequently be read in and used to create a new simulation involving a pathogen-infected host.

New biodistribution simulation and new pathogen-host simulation These wizards guide the user through the process of creating a simulation model containing a host animal and an agent/drug whose distribution through the 
Fig. 5 The BioDMET GUI

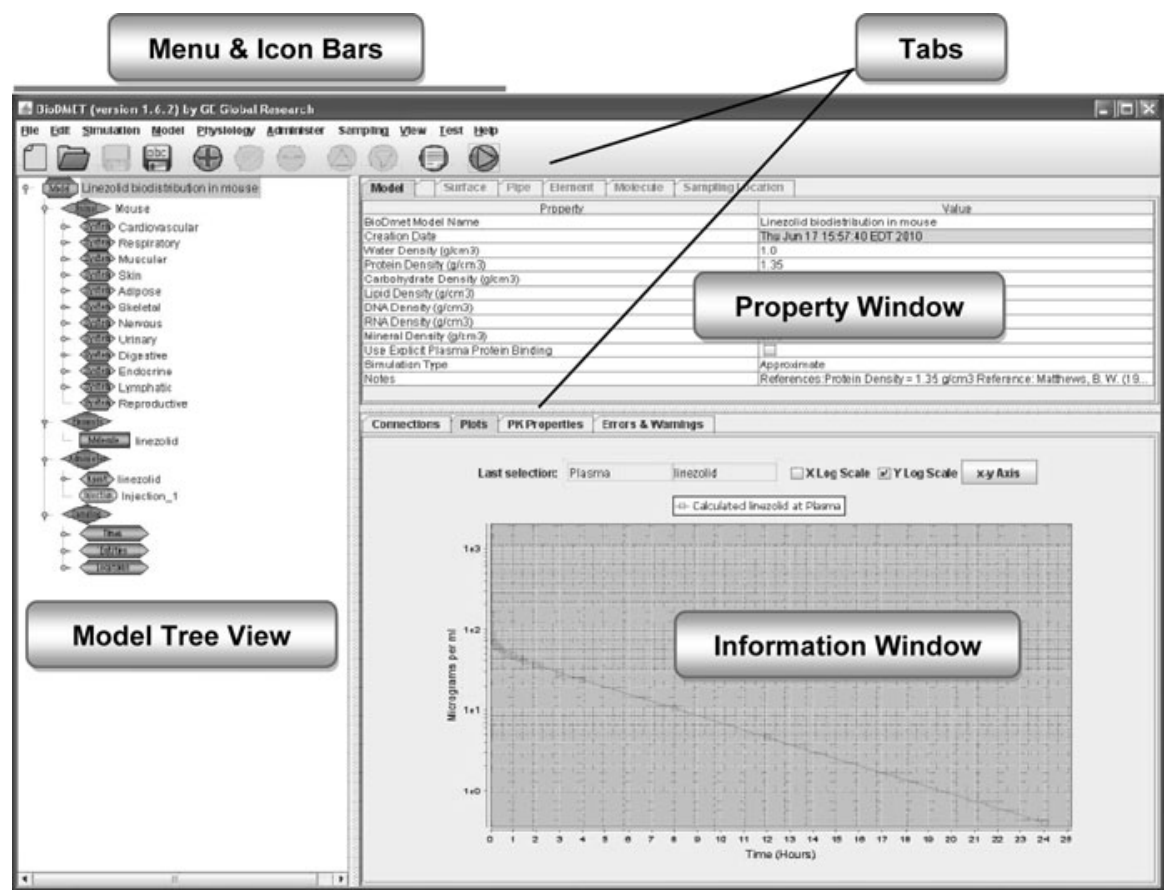

host is to be simulated. In the case of the pathogen-host simulation, a pathogen is added to the system and a pharmacodynamic model is selected for computing the changes in the pathogen load over time.

Sensitivity analysis Disease states are typically characterized by wide inter-patient variability in regard to patient conditions. Sensitivity analysis implemented in BioDMET allows the user to estimate the effect of variations in the model input parameters on the main pharmacokinetic parameters of the agent of interest. This is achieved by performing multiple simulations in which one or multiple input parameters (X-s) are changed and the resulting variations in the output parameters (Y-s) are monitored. The program computes a sensitivity coefficient as the ratio of the change in $\mathrm{Y}$ for a given change in $\mathrm{X}$. This information can then be used to identify key parameters that have major influence on the drug pharmacokinetics and pharmacodynamics. Sensitivity analysis can also provide a quick estimate of how model predictions may change across populations and how drug dosage should be adjusted for achieving the desired effect. The sensitivity analysis wizard guides the user through the selection of input parameters to be varied within user-defined ranges, the type of simulations (varying one or multiple parameters at a time), the number of Monte Carlo steps, and the output parameters to be monitored.

Critically ill patient analysis The BioDMET model parameters were retrieved from the scientific literature and reflect the physiological state of the healthy organism. To account for disease-caused alterations of the normal physiology that can influence the distribution of exogenous agents, we assembled a database of physiology parameters for the critically ill based on literature data and implemented the capability to model several pathological states such as high fever, severe burns, multiple organ failure, etc. Disease states are typically characterized by wide interpatient variability in regard to patient conditions. The Critically Ill Patient Analysis implemented in BioDMET allows the user to estimate the effect of variations in the patient conditions on the main pharmacokinetic parameters of the agent of interest. This information can then be used to adjust the drug dosage to achieve the desired effect. The analysis is analogous to a population study where the individual variations are simulated by Monte Carlo sampling of host parameter values between disease-specific limits. These limits are established based on literature values that are described in the Critically Ill Database available at the BioDMET website under Downloads.

\section{Simulation output}

Once a biodistribution or pathogen-host simulation is completed, the results are displayed in a dialog window arranged in a tabular form. Concentration values for the specified agents are given for each sampling time and location as a percentage of the injected dose. The data can be copied to the clipboard and then pasted into a tool such as MS Excel for performing further analysis. Another way to examine the results is by displaying the concentrationtime curves in the Information Window of the GUI. The 
BioDMET GUI's plotting capability provides an easy way to visualize how concentrations of elements of interest change over time in various tissues and spaces of interest. If more sophisticated visualization and/or in depth analysis of the results is needed, the user can export part of or all the simulation results into a tab-delimited or comma-separated file that can then be imported into another software, such as Excel or Origin. The entire model can be saved in an xml file format defined for the BioDMET application. This file contains both the setup information and the simulation results.

In addition to providing the agent biodistribution data, the following PK parameters are calculated and displayed at the GUI in the Property Window under the PK Properties tab after each simulation: maximum concentration $\left(\mathrm{C}_{\max }\right)$, time when the maximum concentration is achieved $\left(t_{\max }\right)$, area under the curve (AUC) calculated using the trapezoid method from time 0 to the last sampling time point as well as from time 0 to infinity, mean residence time (MRT), half life $\left(t_{1 / 2}\right)$, initial concentration $\left(\mathrm{C}_{0}\right)$, central compartment volume $\left(\mathrm{V}_{\mathrm{c}}\right)$, volume of distribution at steady state $\left(\mathrm{V}_{\mathrm{ss}}\right)$, and clearance $(\mathrm{Cl})$. Except for $\mathrm{C}_{\max }, \mathrm{t}_{\max }$, and $\mathrm{C}_{0}$, the other PK parameters have been derived using a noncompartmental approach [57]. This is based on calculating the AUC of the concentration versus time plot by numerical integration using the trapezoidal rule.

When experimental time-concentration data is present in the model, the above listed PK parameters are derived based on this data as well and are shown under "MEASURED CURVE PARAMETERS" for comparison. A note of caution: even when the experimental data points align closely with the curve representing the calculated drug concentrations, there could be significant discrepancies between the two sets of PK parameters. Most likely, this is due to the fact that experimental data is usually not available for the early time points right after the administration of the agent when drug concentrations change rapidly in time.

After setting up a system with the help of one of the wizards, it is possible to modify the host, pathogen or agent at the GUI. These modified models can be saved as new host, pathogen or agent files in xml format for subsequent simulations.

At the end of each sensitivity analysis or critically ill patient analysis, a window is shown with a summary of the analysis results for a quick assessment. For each monitored output parameter, a bar graph is plotted showing its values for each set of input parameter values sampled. Plots of the sensitivity coefficients of each monitored output relative to the input parameters are also provided after a sensitivity analysis. These summary plots can be saved into a report file in pdf format. This summary is in addition to the text file containing the raw output that is generated for each of these simulations.

\section{Results of testing and validation}

Evaluation of predictive capability

The BioDMET software's ability to predict drug concentrations has been validated using a series of 26 drugs/agents in 44 individual human and animal models. The calculated agent concentrations at various time points were compared to experimentally measured concentrations obtained from the scientific literature. The literature data search was performed to cover multiple host species (human, monkey, guinea pig, rat, mouse) and agent types (drugs, imaging agents) with a wide range of values of pharmacokinetically relevant properties such as molecular weights, $\log P$, and plasma protein binding (Table 1). Even though BioDMET has the capability to model multiple modes of administration (intravenous, intramuscular, subcutaneous, and oral), the majority of the experimental data collected was based on IV administration. A more comprehensive testing and validation of the other administration methods is the focus of a continuing effort.

Good correlation was obtained between experimentally measured and calculated log concentrations of drugs/agents in plasma $\left(\mathrm{R}^{2}=0.93\right)$ and in various other tissues $\left(\mathrm{R}^{2}=0.89\right.$, Fig. 6) following IV administration. The standard deviation of the $\log _{10}$ (measured/calculated) ratios was 0.39 with a mean value of 0.08 for the plasma, and 0.45 with a mean value of 0.13 for the tissues. This level of predictive accuracy is similar to that found in other PBPK models [20]. The individual calculated time-concentration curves compared to the experimental data can be downloaded from the tool website (the link is provided under Validation).

The tool was able to account for the differences in the plasma/muscle and plasma/skin concentration ratios measured for two cephalosporin antibiotics, cefpirome and cefodizime, in healthy volunteers (Fig. 7). This has been attributed mainly to the large differences in plasma protein binding of the two drugs [58]. Similarly, the simulated effect of varying renal function on the plasma concentration of iohexol was in good agreement with the experimentally observed trend (Fig. 8). The validation data (PBPK model parameters, calculated and measured data, including references) is available at the BioDMET web site (http://pdsl.research.ge.com/BioDMET/).

\section{Discussion}

The development of BioDMET has been driven by the need to perform feasibility calculations when faced with complex problems where the interplay of multiple variables can affect the output in a nonlinear fashion. These types of calculations can provide a feel for the magnitude 
Table 1 Summary of model validation data sets collected

\begin{tabular}{|c|c|c|c|c|c|c|c|c|c|}
\hline & Drug or agent & MW & $\log \mathrm{P}$ & PPB (\%) & Human & Rhesus monkey & Guinea pig & Rat & Mouse \\
\hline 1 & 99mTc-DTPA & 491.4 & -6.5 & - & & & & & BioD \\
\hline 2 & Acetaminophen & 151.2 & 0.5 & 20 & $P$ & & & $P$ & \\
\hline 3 & Acyclovir & 225.2 & -1.0 & 33 & $P$ & & & & \\
\hline 4 & Amoxicillin & 365.4 & -4.0 & 20 & $P$ & & & & \\
\hline 5 & Atorvastatin & 558.6 & 5.4 & 98 & & & & $P$ & \\
\hline 6 & Azathioprine & 166.2 & 0.9 & 20 & $P$ & & & & \\
\hline 7 & Caffeine & 194.2 & -0.5 & 30 & $P$ & & & & \\
\hline 8 & Cefodizime & 584.7 & 0.2 & 70 & $P, U, M, S, B$ & & & & \\
\hline 9 & Cefozopran & 516.5 & -3.0 & 8 & $P, U$ & BioD & & BioD & BioD \\
\hline 10 & Cefpirome & 515.6 & -4.1 & 10 & $P, M, S, L$ & & & & \\
\hline 11 & Diazepam & 284.7 & 3.1 & 98.5 & & & & BioD & \\
\hline 12 & Fexofenadine & 501.7 & 0.9 & 65 & & & & $P$ & \\
\hline 13 & Inulin & 6179.4 & -18.0 & - & $P, U$ & & & & \\
\hline 14 & Iohexol & 821.1 & -4.2 & - & $P($ Renal $)$ & & & & \\
\hline 15 & Iopromide & 791.1 & -3.0 & - & $P$ & & & & \\
\hline 16 & Ketoconazole & 531.4 & 4.2 & 99 & & & & $P$ & \\
\hline 17 & Mannitol & 182.2 & -3.7 & - & $P$ & & & & \\
\hline 18 & Midazolam & 325.8 & 3.1 & 97 & $P$ & & & $P$ & \\
\hline 19 & Nateglinide & 317.4 & 4.0 & 98 & $P$ & & & & \\
\hline 20 & Propranolol & 259.3 & 2.6 & 90 & & & & BioD & \\
\hline 21 & SU5416 (Semaxanib) & 238.3 & 3.0 & - & & $P$ & & & \\
\hline 22 & Theophylline & 180.2 & -0.8 & 48 & & & $P$ & & \\
\hline 23 & Troglitazone & 441.5 & 5.5 & 99 & & & & $P$ & \\
\hline 24 & Velcade & 384.2 & 1.5 & 83 & $P$ & & & & \\
\hline 25 & Verapamil & 454.6 & 5.0 & 95 & $P$ & & & $P$ & \\
\hline 26 & Zidovudine (AZT) & 267.2 & -2.4 & 31.5 & & $P$ & & & \\
\hline
\end{tabular}

Italic boxes correspond to existing experimental data. The definition of letter symbols: BioD full biodistribution in all major organ tissues, $B$ bone, $M$ muscle, $L$ lung, $P$ plasma, $S$ skin, $U$ urine, Renal renal function data available

of the challenges before any experimental work has been started. They can also highlight the most problematic areas or the ones where critical data is missing. As more and more experimental data becomes available, the models can be refined to provide more accurate predictions and help explain sometimes unexpected results.

One of the main application areas is assessing the imageability of certain anatomical or disease conditions and delineating the property space of the imaging agent and of the target that could produce images of acceptable quality. The other area is that of drug development. The question here is whether or not a drug candidate could reach the target tissue in high enough concentrations long enough to produce a therapeutic effect while producing minimal toxicity in tissues that are most susceptible.

\section{Establishing imageability criteria}

The system model illustrated in Fig. 9 provides the capability to calculate the expected in vivo clinical images for a set of input properties of the biomarker, imaging agent, and disease state [60]. It is composed of the whole-body PBPK model BioDMET, whole body anatomical maps, and analytical and Monte Carlo image simulator codes for positron emission tomography (PET) and single photon emission computed tomography (SPECT). The input includes anatomical and physiological information about the host and the disease state of interest, physico-chemical as well as biological properties of the agent and its in vivo target. BioDMET calculates the in vivo concentrations of the examined imaging agent in all major body tissues over time, which is used to compute the time-activity curves of a radiolabeled agent. This is then used as the input, along with detailed 3D anatomy phantoms of the human or animal subject, into the physics-based imaging simulator to generate images showing how the potential imaging agent would work. The images are processed just like actual measured images, delineating the regions of interest and quantifying the signal.

The process is run somewhat in reverse during feasibility assessments. Starting with the clinical need for 
Fig. 6 A summary of the BioDMET-calculated concentrations versus the in vivo measured concentrations for the 26 test compounds in human, rat and mouse. Each symbol represents the concentration of one compound at one individual time point. Blue triangles represent concentrations in the plasma, red squares represent concentrations in the various other tissues (muscle, bone, lung, brain, etc.). The time points at which the measurements were made ranged from $1 \mathrm{~min}$ up to $24 \mathrm{~h}$ post injection. A total of 454 plasma and 258 other tissue concentrations following IV administration were included

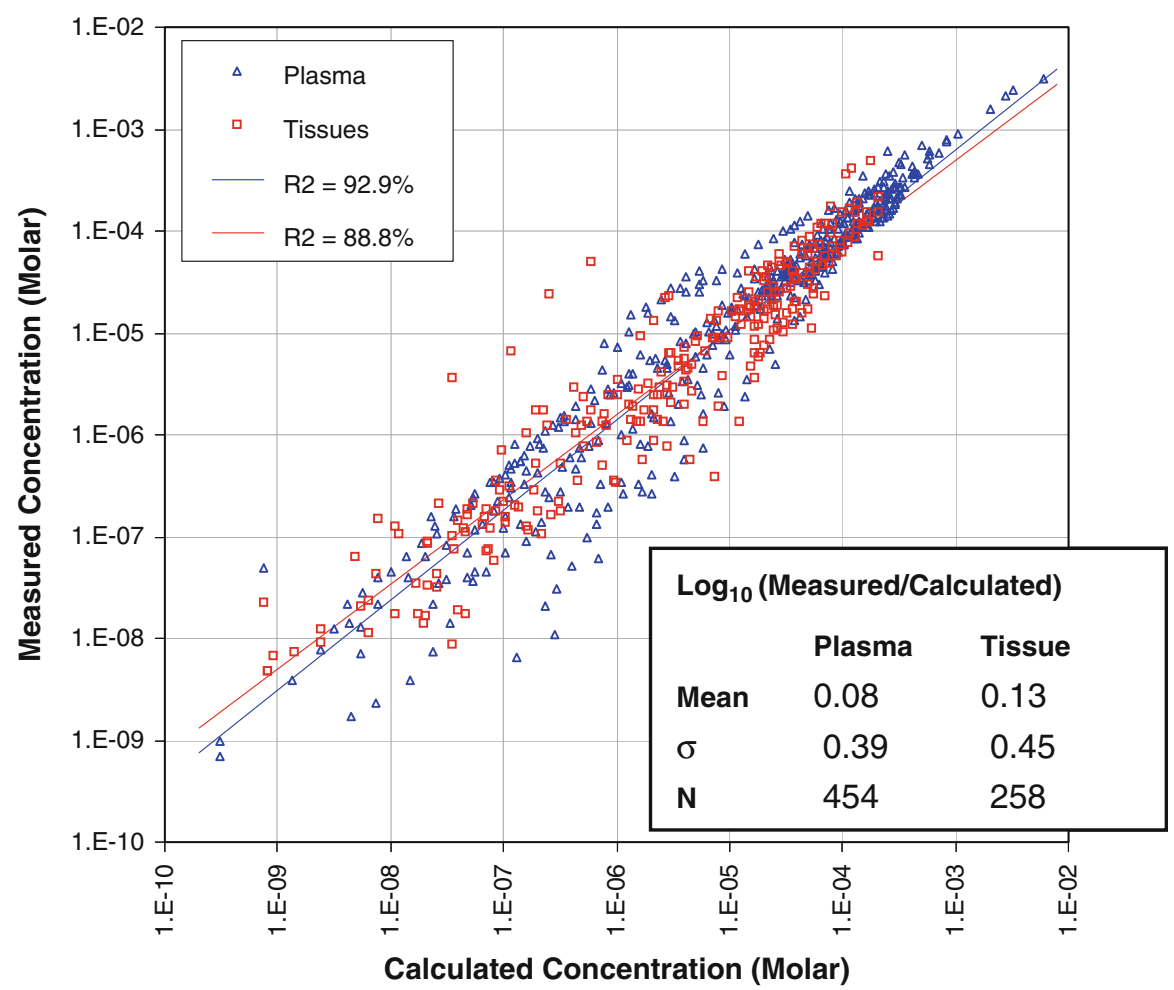

visualizing a specific pathological condition, the properties of the radio-pharmaceutical and target biomarker are derived to meet the imaging requirements for resolution, specificity, and sensitivity given the inherent noise and limitations of an imaging system and protocol. This is accomplished by running multiple calculations with clinically relevant disease and healthy physiology parameters, types and property ranges of the imaging agent and biomarker to be evaluated, as well as the scoring criteria for a good image (e.g., target to background signal at a defined time post injection of the agent). The software then evaluates the performance of different possible combinations of target biomarker and agent biochemical properties against the scoring criteria. Target biomarker properties include its location and concentration levels throughout the body as well as changes with disease progression. Agent properties include molecular weight, $\log P / \operatorname{LogD}$, plasma protein binding, clearance rates, and agent-target binding strength and rate. Imaging requirements such as imaging time post injection and degradation of the signal due to scatter and spillover effects are also considered. In a typical assessment, over 60,000 biodistribution calculations are performed, scored for imaging feasibility, and plotted on color-coded imageability maps (shown in grayscale in Fig. 9). These maps are then used to assess the feasibility of imaging a specific disease for all of the given agenttarget pairs. One example of such a feasibility study was performed to assess the possibility of detecting lesions in the brain of patients with multiple sclerosis using PET imaging [61].

Evaluating drug efficacy and dosing

The main purpose of PBPK modeling is to combine a complex model of an organism with in vitro measurable properties of an agent or drug and predict how it will behave in vivo. This can be taken one step forward by adding further information to the model about the therapeutic and toxic properties of the drug: the effective concentration above which it has measurable therapeutic effects and the toxic concentration at which various toxic effects become detectable. The PBPK model can then be used to estimate the range of doses at which the concentration of the drug in the target tissue can be kept above the effective concentration while keeping it below the toxic levels in tissues that are most prone to damage. This could be more reliable and more specific than the standard in vitro therapeutic index because it can include individualized information about the patient population (age, weight, health status) as well as drug concentrations at target tissues.

\section{Unique features of BioDMET}

Setting up a PBPK model and running a simulation used to be a time-consuming, tedious process that required a large number of parameters to be specified and code writing for 
Fig. 7 Simulated and measured concentrations of cefpirome and cefodizime in human plasma, the interstitial space of muscle, and skin. The experimental data is taken from the published work of Muller et al. [58]
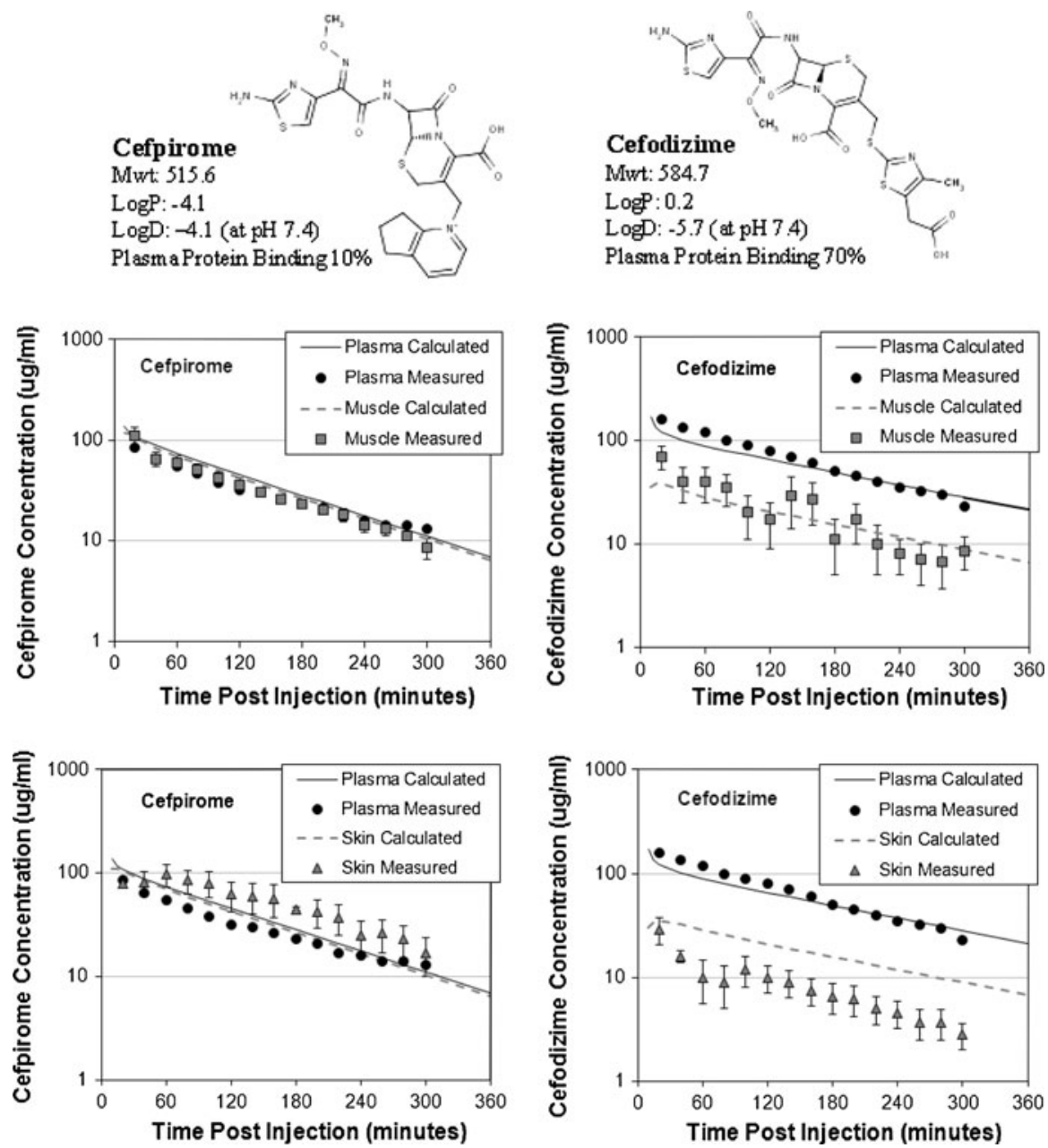

solving differential equations. These types of calculations were accessible typically only to scientists trained in this field. BioDMET is designed to overcome these challenges by providing a scientifically rigorous yet easy-to-use tool with complete, parameterized PBPK models for several species that can be easily customized without the need for programming skills.

The models are comprised of spaces that correspond to real anatomical entities (vasculature, interstitial space, CSF, urine, lymph, subcellular compartments) and are grouped naturally by anatomical organs and tissues. There is no need for artificial compartments that lump together several distinct tissues or organs in order to explain the kinetics of the drug. As a consequence, drug concentrations can be calculated in any of these anatomically relevant spaces and compared to experimentally measured values to validate the model. This also enables the calculation of drug concentration directly at the place of the action for pharmacodynamic evaluations instead of having to use the plasma concentration as a proxy.

Open access to all of the physiology model parameters and rate constants are provided with the human and animal models. References to the model parameters can be accessed through the software GUI under the Notes icon or from the tool documentation provided at the tool's web site under User Guide. A set of experimental data collected from the literature with the corresponding references are also available via the tools web site under Validation. This way the users can experiment with the tools and can run their own testing and validation. Even though the simulations are initiated through a web server, the calculations are done and the results are kept on the user's computer. No data is returned to the server assuring complete data confidentiality.

Customizing/expanding the model

As more and more experimental data becomes available about the mechanisms involved in the biodistribution and action of pharmaceutical agents, the need arises to refine the existing PBPK models by incorporating the new findings. BioDMET was built keeping in mind the need to customize the models for a specific problem and incorporate new knowledge. These modifications can be performed 

measurements for iohexol in human plasma for single patients with different renal functions. Experimental data points were taken from the published work of Gaspari et al. [59]. The GFR varies from healthy function of $115.7 \mathrm{ml} /$ $\mathrm{min} / 1.73 \mathrm{~m}^{2}$ to renal failure rate of $8.9 \mathrm{ml} / \mathrm{min} / 1.73 \mathrm{~m}^{2}$
Fig. 8 Simulations versus
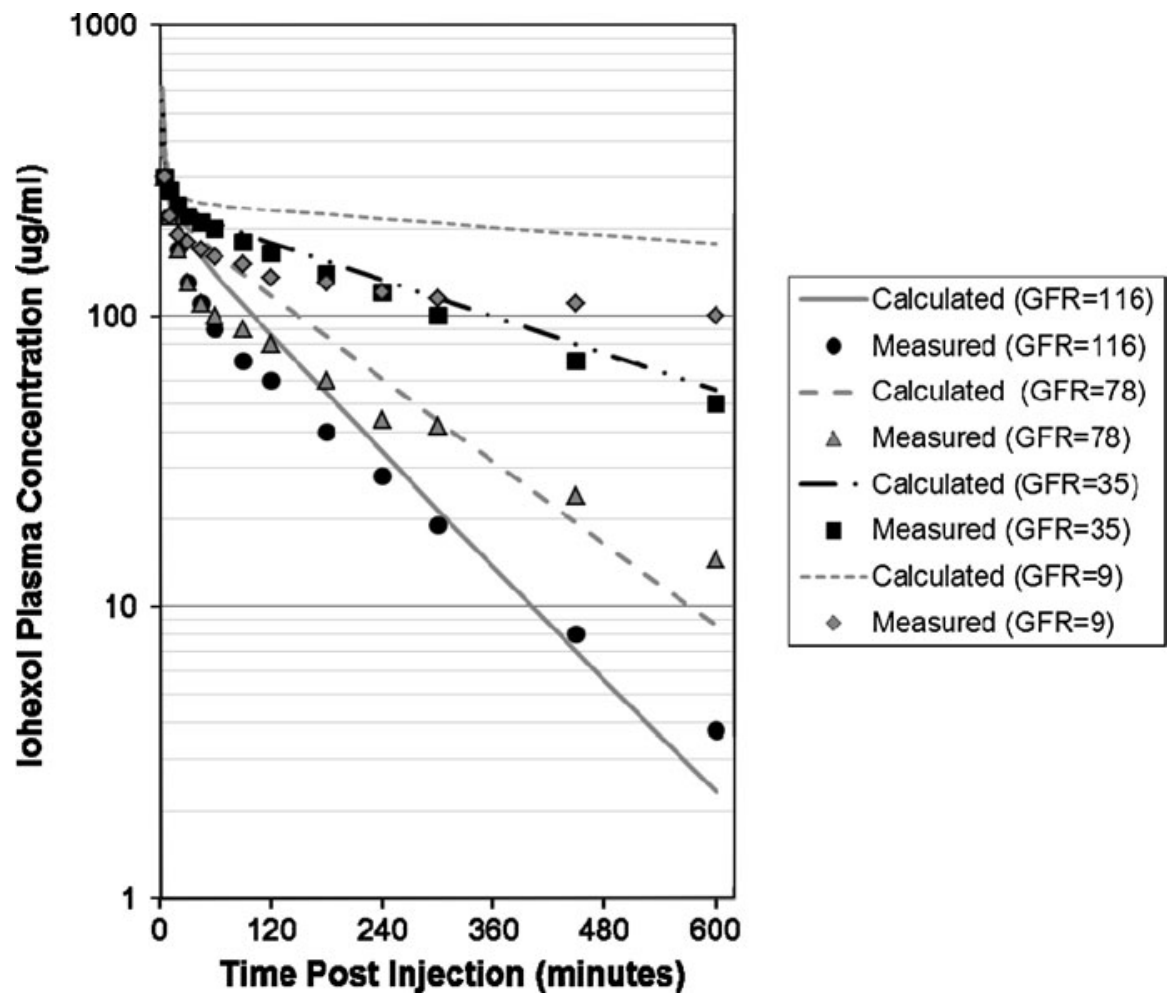

Fig. 9 Computational system model for assessing imaging feasibility. The lighter the color of the box on the imageability map, the higher the probability that the corresponding property combinations lead to successful imaging

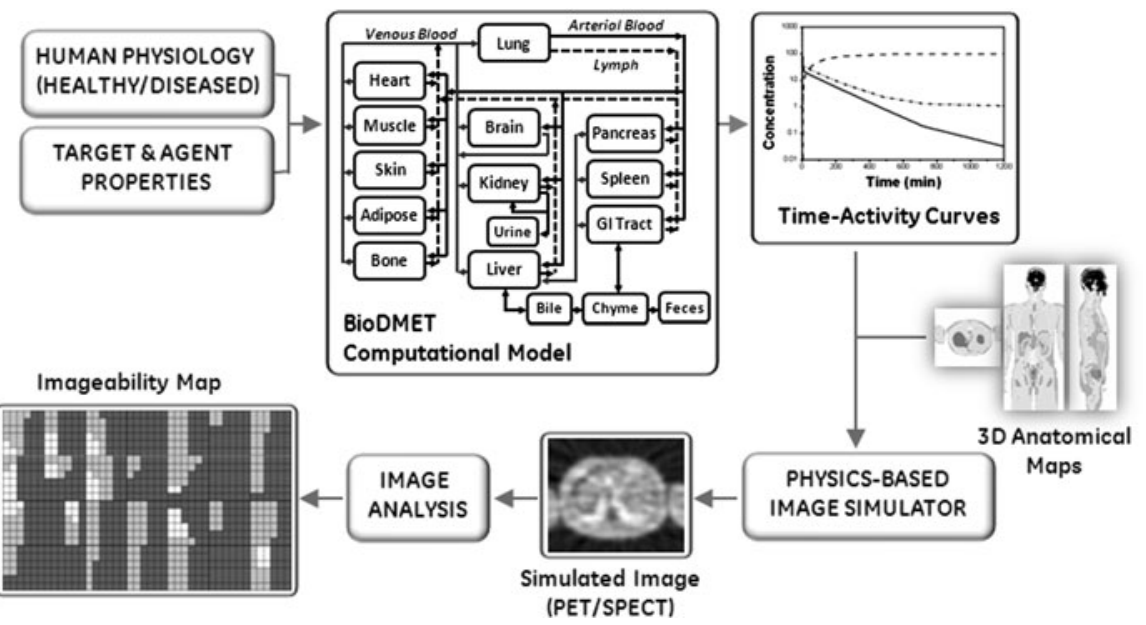

in an intuitive fashion at the GUI without the need to change the code. The customized models can be saved for later use. New spaces can be added or old ones split into multiple components and the parameters characterizing this new space can be changed. New connections can be established between spaces or existing ones can be modified. This is how a tumor, for example, can be inserted into a specific location of a tissue. The host physiology can also be altered to model inter-individual differences or changes caused by disease states. While many of the model parameter values can be changed in an individual fashion, some physiology alterations require a concerted change across multiple organs and tissues because the organism is a closed system and the sum of certain quantities is fixed. For example, tissue inflammation can be modeled by specifying the volume fraction of the inflamed tissue and adjusting the blood and lymph flow, the vascular permeability and change in tissue volume due to edema at a dialog window (Supplementary Fig. S3). After such a change, the tool will make the necessary adjustments to all other spaces connected when the biodistribution of the pharmaceutical is recalculated.

While the pharmacokinetics of some drugs can be modeled quite accurately by passive diffusion and a simple 
one-step liver metabolism, others require a complex interplay of enzymes and transporters. BioDMET allows adding transporters and enzymes and offers two alternatives to describe the process: first order or Michaelis-Menten type equation. The user has to specify the necessary parameters including the location and concentration of the enzyme or transporter, the substrate, the rate of the process, the origin and destination space, the creation and destruction rate of the enzyme, etc. Since the model can track multiple agents and their metabolites, drug-drug interactions can also be taken into account. More details on how to implement such changes are described in the user manual downloadable from the tools' website.

As new therapeutic platforms appear, there is a need for PBPK models to handle peptides, proteins, nucleotides and nanoparticles. It is possible to implement the simulation of these agents in BioDMET. At this point, however, the user has to be aware of the mechanistic differences relative to small molecule drugs that are important for the biodistribution of these molecules, and provide the necessary parameters characterizing the processes involved, including diffusion, transport, metabolism, etc.

Future work is aimed at parameterizing and validating BioDMET for the various therapeutic platforms, including the most frequently encountered transporters into the model, and allowing user-defined equations for describing enzyme- and transporter-mediated processes.

Acknowledgments The authors thank William Jusko, Donald Mager and Chao Xu from the Department of Pharmaceutical Sciences of the University at Buffalo, SUNY, for their independent assessment of BioDMET, the useful discussions, and suggestions for improvements. The authors are grateful for the support of Brion Sarachan (GE Global Research) throughout the development of BioDMET and for the help of Robert Saltzman (GE Global research) in setting up the BioDMET web site. This work has been partially supported by the U.S. Defense Threat Reduction Agency under award number HDTRA1-08-C-0052. However, any opinions, findings, conclusions or other recommendations expressed herein are those of the authors and do not necessarily reflect the views of the U.S. Defense Threat Reduction Agency.

Open Access This article is distributed under the terms of the Creative Commons Attribution Noncommercial License which permits any noncommercial use, distribution, and reproduction in any medium, provided the original author(s) and source are credited.

\section{References}

1. Leahy DE (2004) Drug discovery information integration: virtual humans for pharmacokinetics. Biosilico 2:78-84

2. Lin J, Sahakian DC, de Morais SM, Xu JJ, Polzer RJ, Winter SM (2003) The role of absorption, distribution, metabolism, excretion and toxicity in drug discovery. Curr Top Med Chem 3:1125-1154
3. van de Waterbeemd H, Gifford E (2003) ADMET in silico modelling: towards prediction paradise? Nat Rev Drug Discov 2:192-204

4. Peters SA, Ungell AL, Dolgos H (2009) Physiologically based pharmacokinetic (PBPK) modeling and simulation: applications in lead optimization. Curr Opin Drug Discov Devel 12:509-518

5. EPA. US (2006) Approaches for the application of physiologically based pharmacokinetic (PBPK) models and supporting data in risk assessment (Final report). EPA/600/R-05/043F

6. EPA. US (2006) Use of physiologically based pharmacokinetic (PBPK) models to quantify the impact of human age and interindividual differences in physiology and biochemistry pertinent to risk (Final report). EPA/600/R-06/014A

7. Charnick SB, Kawai R, Nedelman JR, Lemaire M, Niederberger W, Sato H (1995) Perspectives in pharmacokinetics. Physiologically based pharmacokinetic modeling as a tool for drug development. J Pharmacokinet Biopharm 23:217-229

8. Leahy DE (2003) Progress in simulation modelling for pharmacokinetics. Curr Top Med Chem 3:1257-1268

9. Edginton AN, Theil FP, Schmitt W, Willmann S (2008) Whole body physiologically-based pharmacokinetic models: their use in clinical drug development. Expert Opin Drug Metab Toxicol 4:1143-1152

10. Parrott N, Jones H, Paquereau N, Lave T (2005) Application of full physiological models for pharmaceutical drug candidate selection and extrapolation of pharmacokinetics to man. Basic Clin Pharmacol Toxicol 96:193-199

11. Sinskey AJ, Finkelstein SN, Cooper SM (2004) Medical imaging in drug discovery, Part I. PharmaGenomics. March/April:20-25, Advanstar Communications, Edison, NJ

12. Finkelstein SN, Sinskey AJ, Cooper SM (2004) Medical imaging in drug discovery, Part II. PharmaGenomics. May:20-24, Advanstar Communications, Edison, NJ

13. Grass GM, Sinko PJ (2002) Physiologically-based pharmacokinetic simulation modelling. Adv Drug Deliv Rev 54:433-451

14. Jones HM, Gardner IB, Watson KJ (2009) Modelling and PBPK simulation in drug discovery. AAPS J 11:155-166

15. Lave T, Parrott N, Grimm HP, Fleury A, Reddy M (2007) Challenges and opportunities with modelling and simulation in drug discovery and drug development. Xenobiotica 37: $1295-1310$

16. Nestorov I (2007) Whole-body physiologically based pharmacokinetic models. Expert Opin Drug Metab Toxicol 3:235-249

17. Rowland M, Balant L, Peck C (2004) Physiologically based pharmacokinetics in drug development and regulatory science: a workshop report (Georgetown University, Washington, DC, May 29-30, 2002). AAPS PharmSci 6:E6

18. Schmitt W, Willmann S (2004) Physiology-based pharmacokinetic modeling: ready to be used. Drug Discov Today Technol $1: 449-456$

19. Thygesen P, Macheras P, Van Peer A (2009) Physiologicallybased PK/PD modelling of therapeutic macromolecules. Pharm Res 26:2543-2550

20. Parrott N, Paquereau N, Coassolo P, Lave T (2005) An evaluation of the utility of physiologically based models of pharmacokinetics in early drug discovery. J Pharm Sci 94:2327-2343

21. Marx V (2005) Molecular imaging: companies set out to sharpen the in vivo perspective with new machines and novel contrast agents. Chem Eng News 83:25-34

22. Massoud TF, Gambhir SS (2003) Molecular imaging in living subjects: seeing fundamental biological processes in a new light. Genes Dev 17:545-580

23. Barboriak DP, MacFall JR, Viglianti BL, Dewhirst Dvm MW (2008) Comparison of three physiologically-based pharmacokinetic models for the prediction of contrast agent distribution 
measured by dynamic MR imaging. J Magn Reson Imaging 27:1388-1398

24. Mescam M, Kretowski M, Bezy-Wendling J (2010) Multiscale model of liver DCE-MRI towards a better understanding of tumor complexity. IEEE Trans Med Imaging 29:699-707

25. Mescam M, Eliat PA, Fauvel C, Certaines JD, Bezy-Wendling J (2007) A physiologically based pharmacokinetic model of vascular-extravascular exchanges during liver carcinogenesis: application to MRI contrast agents. Contrast Media Mol Imaging 2:215-228

26. Kretowski M, Bezy-Wendling J, Coupe P (2007) Simulation of biphasic CT findings in hepatic cellular carcinoma by a two-level physiological model. IEEE Trans Biomed Eng 54:538-542

27. GastroPlus. http://www.simulations-plus.com/Products.aspx?grpID= $3 \& \mathrm{cID}=16 \& \mathrm{pID}=11$ (accessed 2009)

28. SimCyp. http://www.simcyp.com/ProductServices/Simulator/ (accessed 2009)

29. Willmann S, Lippert J, Sevestre M, Solodenko J, Fois F, Schmitt W (2003) PK-Sim ${ }^{\circledR}$ : a physiologically based pharmacokinetic "whole-body" model. Biosilico 1:121-124

30. PK-Sim. http://www.systems-biology.com/products/pk-sim.html (accessed 2009)

31. MEGen: the PBPK database \& model equation generator. http:// xnet.hsl.gov.uk/megen/ (accessed 2009)

32. JSim. http://www.physiome.org/jsim/ (accessed 2009)

33. Levitt DG (2002) PKQuest: a general physiologically based pharmacokinetic model. introduction and application to propranolol. BMC Clin Pharmacol 2:5

34. Levitt DG (2009) PKQuest_Java: free, interactive physiologically based pharmacokinetic software package and tutorial. BMC Res Notes 2:158

35. SAAM II: simulation, analysis and modeling software for kinetic analysis. http://depts.washington.edu/saam $2 /$ (accessed 2011)

36. acslX Software for Modeling and Simulation. http://www.ac slsim.com/ (accessed 2010)

37. Leahy DE (2006) Integrating in vitro ADMET data through generic physiologically based pharmacokinetic models. Expert Opin Drug Metab Toxicol 2:619-628

38. Brightman FA, Leahy DE, Searle GE, Thomas S (2006) Application of a generic physiologically based pharmacokinetic model to the estimation of xenobiotic levels in rat plasma. Drug Metab Dispos 34:84-93

39. Brightman FA, Leahy DE, Searle GE, Thomas S (2006) Application of a generic physiologically based pharmacokinetic model to the estimation of xenobiotic levels in human plasma. Drug Metab Dispos 34:94-101

40. Cloe ${ }^{\circledR}$ PK. https://www.cloegateway.com/ (accessed 2009)

41. Blancato JN, Power FW, Brown RN, Dary CC (2006) Exposure related rose estimating model (ERDEM) a physiologically-based pharmacokinetic and pharmacodynamic (PBPK/PD) model for assessing human exposure and risk. EPA/600/R-06/061

42. CEMC-PBPK Model. http://www.trentu.ca/academic/aminss/ envmodel/models/PBPK100.html (accessed 2010)

43. Brissova M, Fowler MJ, Nicholson WE, Chu A, Hirshberg B, Harlan DM, Powers AC (2005) Assessment of human pancreatic islet architecture and composition by laser scanning confocal microscopy. J Histochem Cytochem 53:1087-1097

44. Rippe B, Haraldsson B (1994) Transport of macromolecules across microvascular walls: the two-pore theory. Physiol Rev 74:163-219

45. Guyton AC, Hall JE (2005) Medical physiology. Saunders, Philadelphia

46. Lazzara MJ, Deen WM (2007) Model of albumin reabsorption in the proximal tubule. Am J Physiol Renal Physiol 292:F430-F439

47. Birn H, Christensen EI (2006) Renal albumin absorption in physiology and pathology. Kidney Int 69:440-449

48. Houston JB (1994) Utility of in vitro drug metabolism data in predicting in vivo metabolic clearance. Biochem Pharmacol 47:1469-1479

49. Simionescu M, Simionescu N, Palade GE (1974) Morphometric data on the endothelium of blood capillaries. J Cell Biol 60:128-152

50. Takakura Y, Mahato RI, Hashida M (1998) Extravasation of macromolecules. Adv Drug Deliv Rev 34:93-108

51. Lum H, Malik AB (1994) Regulation of vascular endothelial barrier function. Am J Physiol 267:L223-L241

52. Gaumet M, Vargas A, Gurny R, Delie F (2008) Nanoparticles for drug delivery: the need for precision in reporting particle size parameters. Eur J Pharm Biopharm 69:1-9

53. Ballet F (1990) Hepatic circulation: potential for therapeutic intervention. Pharmacol Ther 47:281-328

54. Henderson JR, Moss MC (1985) A morphometric study of the endocrine and exocrine capillaries of the pancreas. Q J Exp Physiol 70:347-356

55. Fujikawa M, Nakao K, Shimizu R, Akamatsu M (2007) QSAR study on permeability of hydrophobic compounds with artificial membranes. Bioorg Med Chem 15:3756-3767

56. Chou C, McLachlan AJ, Rowland M (1995) Membrane permeability and lipophilicity in the isolated perfused rat liver: 5-ethyl barbituric acid and other compounds. J Pharmacol Exp Ther 275:933-940

57. Gibaldi M, Perrier D (1982) Pharmacokinetics. Marcel Dekker, Inc., New York

58. Muller M, Haag O, Burgdorff T, Georgopoulos A, Weninger W, Jansen B, Stanek G, Pehamberger H, Agneter E, Eichler HG (1996) Characterization of peripheral-compartment kinetics of antibiotics by in vivo microdialysis in humans. Antimicrob Agents Chemother 40:2703-2709

59. Gaspari F, Perico N, Ruggenenti P, Mosconi L, Amuchastegui CS, Guerini E, Daina E, Remuzzi G (1995) Plasma clearance of nonradioactive iohexol as a measure of glomerular filtration rate. J Am Soc Nephrol 6:257-263

60. Graf JF, Sarachan BD, Spliker ME (2008) Method and apparatus for assessing feasibility of probes and biomarkers. US Patent Application US20100106423A1, Published 29 Apr 2010; Filed 28 Oct 2008

61. Zavodszky MI, Graf JF, Tan Hehir CA (2011) Feasibility of imaging myelin lesions in multiple sclerosis. Int $\mathrm{J}$ Biomed Imaging 2011:953806 